\title{
La protección ambiental en los procesos de integración. Aportes para cubrir un déficit del Mercosur*
}

\section{Environmental Protection in Integration Processes. Contributions to Cover a Deficit in Mercosur}

\section{Alberto César Moreira*}

SUMARIO: I. La dimensión ambiental en los procesos de integración. II. UNASUR y medio ambiente. III. Mercosur ambiental. Aportes para la creación de un fondo específico. IV. Reflexiones finales. Cursos de acción. V. Bibliografía.

* Artículo recibido el 30 de noviembre de 2010 y aceptado para su publicación el 29 de junio de 2011.

** Docente de la Facultad de Derecho de la Universidad Nacional de Mar del Plata y director del Grupo de Estudio Medio Ambiente de la Sociedad Latinoamericana para el Derecho Internacional. El autor quiere agradecer la colaboración de la doctora Mirta Laciar, coordinadora nacional del Subgrupo de Trabajo 6 Medio Ambiente del Mercosur por sus valiosos aportes y comentarios. Las opiniones vertidas en este trabajo, así como cualquier error que pudiera existir, son de exclusiva responsabilidad del autor. 
RESUMEN: El presente trabajo tiene como punto de partida considerar que la protección ambiental es una dimensión clave en todo proceso de integración regional. Esta idea motora invita a introducirnos en el espacio de convergencia que ofrece la UNASUR y, en particular, en uno de sus bloques, el Mercosur. Se exploran las oportunidades que un fondo de implementación de planes y proyectos ambientales brindaría y se señalan los desarrollos más significativos alcanzados en vista a su constitución; en especial, el perfil y funcionamiento del Fondo para la Convergencia Estructural del Mercosur. Finalmente, se brindan propuestas para crear un instrumento de financiamiento que traduzca en acciones concretas la dimensión ambiental de este espacio de integración.

Palabras clave: UNASUR, Mercosur, protección ambiental, FOCEM, Fondo Ambiental de Implementación.

ABSTRACT: This paper is a starting point to consider that environmental protection is a key dimension in any regional integration process. The driving idea invites us into the convergence space offered by the USAN, and in particular, in one of its components, Mercosur. It explores the opportunities that a Fund for the Implementation of environmental plans and projects would provide and identifies the most significant developments made in view of its constitution, in particular, the profile and performance of the Fund for Structural Convergence of Mercosur. Finally, there are proposals to create a financing instrument which would translate into concrete actions the environmental dimension of this space integration.

Descriptors: USAN, Mercosur, Environmental Protection, FOCEM, Implementation Environmental Fund.

RÉSUMÉ: Ce document est un point de départ pour considérer que la protection de l'environnement est une dimension essentielle dans tout processus d'intégration régionale. L'idée motrice nous invite dans l'espace de la convergence offerte par l'UNASUR, et particulièrement, dans un de ses blocs, le Mercosur. Il explore les possibilités de créer un Fonds pour la mise en ceuvre des plans et des projets environnementaux de fournir et identifie les développements les plus importants réalisés en vue de sa constitution, notamment, le profil et le rendement du Fonds de convergence structurelle du MERCOSUR. Finalement, il y a des propositions visant à créer un instrument de financement qui traduite en actions concrètes la dimension environnementale de cette intégration de l'espace.

Mots-Clés: UNASUR, Mercosur, Protection de l'environnement, FOCEM, Fonds pour l'environnement. 
La protección ambiental debe ser una de las dimensiones clave en todo proceso de cooperación e integración regional, ya que no es posible alcanzar un desarrollo económico sustentable si no se diseñan e implementan las medidas de tutela del ambiente. ${ }^{1}$

A partir de un enfoque sistémico u holístico del medio ambiente y del paradigma del desarrollo sustentable, es imposible soslayar la estrecha relación que existe entre los factores comerciales, económicos y sociales con la protección del ambiente. ${ }^{2}$

Así, procesos como el Tratado de Libre Comercio de América del Norte, ${ }^{3}$ la Unión Europea (UE), la Comunidad Andina de Naciones (CAN) y el Mercado Común del Sur (Mercosur), entre otros, exhiben un nuevo tratamiento de las cuestiones y los problemas ambientales, de acuerdo con los objetivos de cada uno de ellos y el estado de profundiza-

1 CEPAL, El Desarrollo Sustentable: Transformación productiva, equidad y medio ambiente, Santiago de Chile, 1991, p. 109: "Para poner en práctica el desarrollo sustentable se requiere de una transformación estructural del sistema social en general pero, especialmente, del sistema económico, pues uno de los objetivos inmediatos para revertir las tendencias de insostenibilidad es "producir más y mejor" en lo que se refiere a la utilización de recursos y energías; mejorar la eficiencia de los procesos productivos (reciclado, reutilización) y especialmente reformar la base tecnológica actual para que impulse una actividad económica que no sea ambientalmente destructiva sino creativa."

2 "La relación entre comercio internacional y medio ambiente es compleja y de mutuo impacto. En general, se diferencian, por un lado, los posibles efectos de la liberalización comercial sobre la calidad del ambiente y sobre las políticas de protección ambiental y, por el otro, el impacto de las regulaciones y políticas ambientales en la dinámica del comercio internacional. Planteado en sus términos más básicos, la tensión entre liberalización comercial y protección ambiental podría graficarse como un conflicto entre lógicas contradictorias: la lógica de la liberalización comercial que consiste, fundamentalmente, en la promoción del intercambio comercial mediante la remoción de las barreras, arancelarias y no arancelarias que gravan el comercio entre las naciones; y por el otro, la lógica de la política ambiental que requiere de un "hacer" por parte de los Estados para proteger la calidad del ambiente, ya sea mediante regulaciones o incentivos". Ryan, D., Mercosur y ambiente, "Ambiente, Derecho y Sustentabilidad", Buenos Aires, La Ley, 2000, p.383.

3 The North American Free Trade Agreement (NAFTA). “... El texto del tratado contiene numerosas y significativas disposiciones que regulan las tensiones propias de la liberalización comercial y la protección ambiental, y en este sentido, el NAFTA ha sido descripto como el “más ambiental de todos los acuerdos tradicionales de comercio”. Ryan, D., op. cit., p. 386. 
ción alcanzado. En mayor o menor medida, las políticas medioambientales se van introduciendo como un contenido transversal en el diseño de las otras políticas (económica, social, cultural, de empleo, etcétera).

En el caso de la Unión Europea, máximo referente en materia de integración, la política ambiental reviste carácter estructural ${ }^{4}$ y sus exigencias se encuentran incorporadas a las demás políticas y acciones, ${ }^{5}$ siendo, por otra parte, una de las prioridades para la cofinanciación comunitaria. ${ }^{6}$

Por su parte, resulta innegable la dimensión ambiental del proceso Mercosur, ${ }^{7}$ así como su consolidación en la estructura institucional, aspectos que abordaremos más adelante. No obstante, se observa una gran dificultad para avanzar en cuestiones sustanciales, lo que demuestra que la protección del ambiente continúa ocupando un lugar periférico en las prioridades de los Estados que integran este espacio.

4 El Acta Única Europea del 16 de febrero de 1986 incorporó al Tratado de la Comunidad el Título VII relativo al medio ambiente. Posteriormente, el tratado de Maastricht (1992) jerarquizó la política ambiental elevándola a un lugar central dentro de los objetivos de la Unión. Para un estudio profundo del derecho ambiental en la Unión Europea véase Plaza Martín, C., Derecho Ambiental de la Unión Europea, Madrid, Tirant Lo Blanch, 2005.

5 "La U.E. es un fenómeno único de la política internacional ... la temática ambiental es una de las áreas más dinámicas del accionar de la U.E.; de acuerdo a Konrad von Moltke, actualmente no queda casi área de la gestión ambiental de los Estados miembros que estén al margen de la legislación de la U.E ; en un primer momento, era posible identificar argumentos de carácter económico para justificar este accionar, hoy, la protección y la promoción de la calidad ambiental se plantean como objetivos en sí mismos del accionar de la U.E.”. Ryan, D., op. cit., p. 386.

6 La Unión Europea ha instrumentado sus políticas de protección ambiental a través de los "Programas de Acción Comunitario en Materia de Medio Ambiente" (PMA). El último de ellos, que abarca un período de diez años que se inicia el 22 de julio de 2002, define las prioridades y objetivos de la política del medio ambiente europeo y detalla las medidas que deben adoptarse para la implementación de sus estrategia en materia de desarrollo sostenible. Véase Decisión núm. 1600/2002 / CE del Parlamento Europeo y del Consejo, de 22 de julio de 2002, por la que se establece el Sexto Programa de Acción Comunitario en Materia de Medio Ambiente. Diario Oficial núm. L 242 de 10/09/2002.

7 "La protección del medio ambiente informa de manera central el proceso integrador del Mercosur". Primera conclusión del XIX Congreso Argentino de Derecho Internacional de la Asociación Argentina de Derecho Internacional, Córdoba, 2009. 
También la CAN aborda la cuestión ambiental: cuenta con un instrumento de carácter marco, una agenda que atiende ciertos ejes estratégi$\cos ^{8}$ y un organigrama institucional desarrollado.

Lamentablemente, en ninguno de los casos se ha creado un Fondo específico, al modo del Instrumento Financiero para el Medio Ambiente de la Unión Europea (LIFE), ${ }^{9}$ que transfiera recursos para financiar proyectos de implementación de políticas, planes y proyectos ambientales.

\section{UNASUR Y MEDIO AMBIENTE}

La Unión de Naciones Suramericanas (Unasur), constituida formalmente a través del Tratado suscripto en Brasilia en 2008, no es una agenda exclusivamente de tipo comercial. Muy por el contrario, ya en el preámbulo del acta fundacional, los Estados miembros afirman su determinación de construir una identidad y ciudadanía suramericanas y desarrollar un espacio regional integrado en lo político, económico, social, cultural, ambiental, energético y de infraestructura, para contribuir al fortalecimiento de la unidad de América Latina y el Caribe. Lo ambicioso y amplio del proyecto se refleja en el extenso listado de objetivos específicos que contiene el artículo 3o. del Tratado Constitutivo. ${ }^{10}$

8 En el marco de la Agenda Ambiental Andina (2006-2010) se implementan la Estrategia Regional de Biodiversidad para los Países del Trópico Andino (ERB) y la Estrategia Andina para la Prevención y Atención de Desastres (EAPAD), y se trabaja en la formulación de las estrategias regionales de cambio climático y de recursos hídricos, con el fin de apoyar la políticas ambientales nacionales y de fomentar su armonización.

9 El Instrumento LIFE financia proyectos desde 1992 y ha transitado cuatro fases. El actual programa es el LIFE+, cuyo período va del 1 de enero 2007 al 31 de diciembre de 2013, véase Reglamento (CE) núm. 614/2007 del Parlamento Europeo y del Consejo, de 23 de mayo de 2007, relativo al instrumento financiero para el medio ambiente (LIFE+). Diario Oficial, núm. L149, del 9 de junio de 2007.

10 "La Unasur no pretende ser una mera agenda comercial habida cuenta que incluye también cuestiones relativas a inversión, cooperación y diálogo político. Aunque para lograr estos fines no tiene por qué faltarle empuje económico si se tienen en cuenta los indicadores macroeconómicos y de otra naturaleza que caracterizan a la región sudamericana”. Cienfuegos, Mateo M., ¿Es la Unión de Naciones Sudamericana un proyecto viable? en Mercosur y Unión Europea, Córdoba, Lerner, 2008, p. 113. 
En lo que se refiere a la protección ambiental, queda expresamente consagrada en el preámbulo y en varias de las disposiciones del Tratado de Brasilia, lo que se explica por un escenario natural que posee, entre otros recursos, el 27\% del agua dulce del mundo, importantísimas reservas de hidrocarburos, ocho millones de kilómetros cuadrados de bosque y dos océanos que bañan sus costas. ${ }^{11}$

Dentro de los objetivos de la Unasur se incluye: "La protección de la biodiversidad, los recursos hídricos y los ecosistemas, así como la cooperación en la prevención de las catástrofes y en la lucha contra las causas y los efectos de cambio climático". ${ }^{12}$

A poco de ahondar en este proceso regional, se advierte que la extensión y variedad de los fines buscados no se corresponden con la estructura institucional creada, tanto en lo que se refiere a la cantidad de los órganos, como a su composición (siempre de carácter intergubernamental), y cuyas decisiones no resultan aplicables de manera directa. Queda planteada la incógnita: ¿cómo podrán cumplirse esos objetivos? ${ }^{13}$

Debemos admitir, no obstante, que no se parte de cero, ya que una de las estrategias planteadas desde la génesis de la Unasur es aprovechar el desarrollo e instituciones logrados en los dos procesos de integración regional que convergen, el Mercosur y la CAN, tarea que no parece sencilla.

\section{Convergencia y profundización}

En la Declaración de Cusco del 8 de diciembre de $2004{ }^{14}$ se afirmó que: "La Comunidad Sudamericana de Naciones establecerá e implementará

11 Véase http://www.comunidadandina.org/sudamerica.htm.

12 Si bien el orden en la enumeración de los objetivos no atribuye jerarquía, no es un dato menor que la cuestión ambiental figure en el séptimo lugar, incluso mucho antes de la cooperación económica y comercial.

13 "El funcionamiento adecuado de un proceso de integración requiere la instauración de un sistema institucional flexible en su funcionamiento y con capacidad de aprobar normas comunes obligatorias, que sean aplicables directamente a sus países miembros y sus ciudadanos y disfruten de primacía sobre las normas nacionales que las contradigan...”. Cienfuegos, Mateo M., op. cit., p. 112.

14 La Declaración de Cusco sobre la Comunidad Sudamericana de Naciones fue aprobada en la III Cumbre Presidencial Sudamericana, que decidiera la creación de la Comunidad Sudamericana de Naciones (Casa), hoy Unasur. 
progresivamente sus niveles y ámbitos de acción conjunta, promoviendo la convergencia y sobre la base de la institucionalidad existente, evitando la duplicación y superposición de esfuerzos...”.

En esa dirección, en la primera reunión de jefes de Estado (Brasilia, 2005) de la entonces Comunidad Sudamericana de Naciones, se identificó al medio ambiente como una de las áreas prioritarias de la Comunidad y se encomendó a las secretarías de los procesos de integración involucrados un Informe sobre cuestiones jurídicas e institucionales. El análisis comparativo que se elaboró ${ }^{15}$ da cuenta de los marcos normativos e institucionales alcanzados por cada uno de los procesos convergentes. Para los fines de este trabajo, basta adelantar que tanto la CAN como el Mercosur cuentan con un instrumento marco sobre medio ambiente y una estructura institucional que incluye órganos políticos y técnicos.

Hemos anticipado que en ninguno de los esquemas existe un Fondo Ambiental de Implementación, ${ }^{16}$ instrumento que consideramos de suma utilidad para llenar de contenido esta dimensión clave.

Si la Unasur se construirá sobre la base de la convergencia entre la CAN y el Mercosur, quiere decir que cada uno de estos bloques deberá aportar sus fortalezas y, al mismo tiempo, profundizar sus respectivos procesos de integración. ${ }^{17}$

En esa línea, habremos de referirnos sucintamente a los desarrollos verificados en el Mercosur, avances que nos permiten afirmar que el

15 Documento elaborado conjuntamente por las Secretarías de la ALADI, CAN y Mercosur, "Cuestiones Jurídico - Institucionales de la Comunidad Sudamericana“y en particular su Capítulo 1 titulado “Aspectos Normativos e Institucionales en las Áreas Prioritarias de la Comunidad Sudamericana de Naciones”. Véase http://www.comunidadandina.org/csn/estudios. htm.

${ }^{16}$ En términos generales, los Fondos Ambientales de Implementación tienen por objeto permitir la puesta en práctica de las políticas, planes y proyectos ambientales en los distintos niveles de gestión, a través de la transferencia de recursos financieros y de tecnología. Dichos instrumentos pueden ser nacionales o internacionales (de alcance regional, comunitario o universal). Los Fondos internacionales, que suelen coordinarse con otras herramientas de financiamiento, posibilitan el cumplimiento de los compromisos asumidos por los Estados, para alcanzar los objetivos de los Convenios Internacionales de alcance global. Esto permite transformar las iniciativas nacionales para que reporten beneficios a escala regional y/o global.

17 Grajate, R., "Hacia la construcción de la UNASUR. Institucionalidad y Convergencia", MERCOSUR y Unión Europea, Córdoba, Lerner Editora SRL, 2008, p. 145. 
mismo dispone de un marco jurídico apropiado para crear un fondo ambiental, tiene órganos competentes y capacitados para la formulación de esta iniciativa y, fundamentalmente, cuenta con la experiencia de funcionamiento de su primer fondo estructural.

\section{MERCOSUR AMBIENTAL. APORTES PARA LA CREACIÓN DE UN FONDO ESPECÍFICO}

ElTratado de Asunción en ninguno de sus artículos se refiere de manera expresa a la protección del medio ambiente, no obstante figura en el tercer párrafo del preámbulo: ${ }^{18}$

... ese objetivo debe ser alcanzado mediante el más eficaz aprovechamiento de los recursos disponibles, la preservación del medio ambiente, el mejoramiento de las interconexiones físicas, la coordinación de las políticas macroeconómicas y la complementación de diferentes sectores de la economía, con base en los principios de gradualidad, flexibilidad y equilibrio.

El carácter programático del Tratado fundacional permitió que la dimensión ambiental del Mercosur se fuera desarrollando de manera paulatina, inicialmente a través de la Reunión Especializada de Medio Ambiente (REMA).

El trabajo de la REMA se centró en la detección de las asimetrías políticas y legislativas que podían afectar la competitividad. Se buscó establecer una relación horizontal con los distintos subgrupos de trabajo del Grupo Mercado Común. Los temas tratados en las diversas reuniones fueron: el análisis de la legislación vigente en materia ambiental en los Estados parte, las propuestas de directrices para

18 Laciar, M., Medio ambiente y desarrollo sustentable. Los desafíos del Mercosur, Buenos AiresMadrid, Ciudad Argentina, 2003, p. 27: "Su incorporación formal en un tratado de carácter comercial, permite reconocer que sus negociadores no quedaron al margen de las tendencias que se estaban gestando en procesos similares". 
la protección del medio ambiente y concertación de las políticas ambientales, las restricciones no arancelarias de carácter ambiental. ${ }^{19}$

Pero la jerarquización institucional de esta temática se produjo con la conversión de la REMA en el Subgrupo de Trabajo 6 Medio Ambiente (SGT6), ${ }^{20}$ estableciéndose como tareas prioritarias: las restricciones no arancelarias, la competitividad y medio ambiente, las normas internacionales ISO 14.000, los temas sectoriales, el proyecto de instrumento jurídico de medio ambiente en el Mercosur, el sistema de información ambiental y el sello verde Mercosur. El SGT6 fue confirmado en la estructura institucional por Dec. 26/00 del Consejo Mercado Común (CMC) referida al "Relanzamiento del Mercosur".

Si bien hoy puede afirmarse que la política ambiental reviste carácter estructural, la ausencia de un mecanismo de financiamiento específico para implementar programas y proyectos ${ }^{21}$ puede verse como un déficit institucional.

Reconocemos que las propuestas de este tipo de instrumentos generan reparos, no sólo porque requieren un esfuerzo financiero de los Estados sostenido en el tiempo, sino, además, por las numerosas críticas de las que son objeto los fondos internacionales existentes, muchas de ellas atendibles. No obstante ello, en los últimos años se observa un crecimiento cuantitativo y cualitativo de estos mecanismos de financiamiento, en todos los niveles de gestión (nacional, regional, comunitario, universal), y su consolidación como herramientas fundamentales para una práctica del desarrollo sustentable.

Naturalmente, no desconocemos la trascendencia de los fondos de carácter universal, en particular el Fondo para el Medio Ambiente

19 Devia, L., "La política ambiental en el marco del Tratado de Asunción”, Mercosur y Medio Ambiente, Buenos Aires, Ciudad Argentina, 2000, p. 30.

20 Mediante la resolución 10/94 GMC se aprobaron las directrices básicas en materia de política ambiental. Posteriormente, sobre pautas de negociación de los subgrupos de trabajo, reuniones especializadas y grupos ad-hoc, se emitió la resolución 38/95 GMC, en la que se determinó la conversión de la REMA en el SGT6 Medio Ambiente.

21 Vernetti señala que si bien el Mercosur no cuenta aún con un Fondo de esta naturaleza a nivel comunitario (refiriéndose a un mecanismo al modelo del LIFE europeo), detalla los que existen a nivel nacional en tres de los países del bloque. Vernetti, A., "Medio ambiente: Los Fondos Ambientales como mecanismo financiero de recomposición“, Revista Doctrina Judicial, Buenos Aires, 2001-3, pp. 283 y 284. 
Mundial, pero si los comparamos con los instrumentos regionales, estos últimos ofrecen ciertas ventajas. En primer lugar, partimos de una voluntad política de integración y una posición geoestratégica concurrente, al menos en algunos aspectos, por lo que resultará más sencillo determinar las prioridades ambientales desde una mirada nacional y regional. Es de esperar, además, mayor agilidad en la toma de decisiones y laposibilidad de alcanzar el consenso requerido en este tipo de mecanismos. Se agrega, también, que el manejo de la cartera de proyectos es más simple, no sólo por la cantidad de éstos y el volumen de recursos movilizados, sino también por la mayor proximidad de los órganos decisorios con los beneficiarios. Finalmente, será posible una mejor articulación con los esfuerzos nacionales, a modo de obtener sinergias y un mayor impacto a futuro.

Pero además de los beneficios estrictamente ambientales, estos fondos pueden reportar avances en el propio proceso integracionista, ya que contribuyen a la reducción de las asimetrías de los Estados y a la armonización de sus legislaciones, permiten el cumplimiento conjunto de compromisos internacionales, operan como catalizadores de otras fuentes de financiamiento extra bloque y acercan a los distintos actores. En síntesis, profundizan el proceso en marcha.

En el Mercosur se han dado pasos significativos, algunos de ellos fruto de la activa labor del SGT6.

Entre esos avances podemos destacar la aprobación del Acuerdo Marco sobre Medio Ambiente ${ }^{22}$ y de la Política de Promoción y Cooperación en Producción y Consumo Sostenibles, ${ }^{23}$ así como las primeras experiencias de cooperación internacional ambiental. ${ }^{24}$

Pero el hecho más trascendente es, sin duda, el establecimiento del Fondo para la Convergencia Estructural del Mercosur (Focem), por la base jurídica y arquitectura institucional que podría ofrecer, así como por las lecciones que pueden extraerse de su funcionamiento.

22 Aprobado mediante Res. 02/01 del GMC.

23 Dec. 26/07 del CMC.

${ }^{24}$ Si bien no está directamente relacionado con el objeto de esta intervención, otro avance significativo en la materia fue la aprobación del Protocolo Adicional al Acuerdo Marco de Medio Ambiente en materia de Cooperación y Asistencia ante Emergencias Ambientales (Dec. 14/04 del CMC). 
Laciar señala que la adopción del Acuerdo Marco sobre Medio Ambiente no fue fácil y reconoce como antecedente al Proyecto de Protocolo Adicional al Tratado de Asunción elaborado por el SGT6 que no llegó a aprobarse. ${ }^{25}$

Se trata de una norma de carácter blando que no fija obligaciones concretas, plazos, ni responsabilidades y que tiene como eje central la cooperación de los Estados en materia ambiental. ${ }^{26}$

En ese sentido, y en lo que importa al objeto de esta intervención, cabe destacar el Capítulo III del Acuerdo y en particular el artículo 6o., ya que contiene una lista de acciones que los Estados deben adoptar, destacando entre ellas las siguientes:

d) identificar fuentes de financiamiento para el desarrollo de las capacidades de los Estados Parte, a efectos de contribuir con la implementación del presente acuerdo.

i) promover el uso de instrumentos económicos de apoyo a la ejecución de las políticas para la promoción del desarrollo sustentable y la protección del medio ambiente.

Dentro de este marco, la cooperación internacional constituye uno de los mayores desafíos para el SGT6, órgano que ha propuesto instrumentos y explorado canales de cooperación técnica a través del Comité de Cooperación Técnica del Mercosur (CCT).

25 Para analizar el proceso de negociación y adopción del documento véase Laciar, M., op. cit., pp. 89 y ss.

${ }_{26}$ Cfr. artículo 5o. del Acuerdo Marco: "Los Estados Partes cooperarán en el cumplimiento de los acuerdos internacionales que contemplen materia ambiental de los cuales sean parte. Esta cooperación podrá incluir, cuando se estime conveniente, la adopción de políticas comunes para la protección del medio ambiente, la conservación de los recursos naturales, la promoción del desarrollo sustentable, la presentación de comunicaciones conjuntas sobre temas de interés común y el intercambio de información sobre las posiciones nacionales en foros ambientales internacionales". 
A. Una experiencia de cooperación exitosa

La primera experiencia de cooperación en materia ambiental en el ámbito del Mercosur fue el acuerdo: "Fomento de Gestión Ambiental y de Producción Más Limpia (Proyecto CyMA Competitividad y Medio Ambiente)". ${ }^{27}$

Dicho proyecto contribuyó a la instalación de la temática en el sector productivo del Mercosur, siendo su objetivo apoyar a las pequeñas y medianas empresas para que mejoraran su competitividad, a través de una gestión ambiental adecuada y de métodos de producción más limpia y eficiente.

La responsabilidad de la ejecución estuvo a cargo del SGT6, en colaboración con los organismos ambientales nacionales de los Estados parte del Mercosur. El proyecto trabajó en dos planos - a nivel Mercosur y en forma directa con los Estados miembros-- y contó con una primera fase de orientación de dos años (2002-2004) y una segunda de implementación que completó los cinco años de vigencia (2004-2007).

Para llevar a cabo este proyecto fue necesario definir una estructura institucional, por lo que se nombraron puntos focales en cada país y se crearon comités operativos.

El proyecto CyMA proporcionó beneficios tanto a nivel nacional 28 como en el plano regional. En este último escenario, contribuyó a fortalecer el funcionamiento del SGT6 y su articulación con otros actores clave, como el SGT7 Industria, ${ }^{29}$ y ofreció un ejercicio práctico

27 Acuerdo de cooperación técnica concluido entre el Mercosur y el gobierno alemán, a través de la Deustche Gesellschaft für Technische Zusammenarbeit, aprobado mediante Dec. 02/02 del CMC y concluido en 2007.

28 En ese sentido puede verse: "Gestión ambiental y producción más limpia en el Mercosur. Proyecto "Competitividad y Medio Ambiente" en la Argentina. Cinco años con la Cooperación Alemana”, publicación de la Secretaría de Ambiente y Desarrollo Sustentable de la Nación Argentina y de GTZ Deustche Gesellschaft für Technische Zusammenarbeit.

29 En el proyecto se dio especial énfasis a la cadena de valor madera-muebles, en el marco de acciones de cooperación entre los Subgrupos de Trabajo 6 y 7, para contribuir a la implementación del "Programa Foros de Competitividad del Mercosur". En este contexto, el CMC aprobó la Dec. 14/06 sobre "Complementación del Programa Foros de Competitividad por Directrices de Gestión Ambiental y Producción Más Limpia”, que fue promovida por el SGT6 y la Reunión de Ministros de Medio Ambiente. 
de desarrollo de proyecto de cooperación. Se evidenciaron, además, avances orientados al desarrollo de instrumentos de política, siendo un elemento fundamental para la elaboración de la Política de Promoción y Cooperación en Producción y Consumo Sostenibles.

\section{B. La Política de Promoción y Cooperación en Producción y Consumo Sostenibles}

En noviembre de 2005 el SGT6 elaboró una propuesta de Política de Gestión Ambiental y Producción Más Limpia, cuyo Anexo contiene un listado no taxativo de instrumentos de implementación y aplicación. Entre las medidas sugeridas se encuentran la creación del Fondo Mercosur a la Producción Sustentable y de un Fondo ambiental.

Esta propuesta fue parcialmente recogida en el documento aprobado por el CMC: "Política de Promoción y Cooperación en Producción y Consumo Sostenibles en el Mercosur". ${ }^{30}$ La implementación de esta normativa de carácter regional permite avanzar en la temática de manera coordinada entre los Estados parte, constituyendo también un marco de orientación para los trabajos que se desarrollen a nivel nacional.

De acuerdo con el artículo 10 de esta Política, los Estados promoverán la negociación de instrumentos de promoción y cooperación, los cuales se incluyen en el Apéndice I, cuyo inciso $b$ se refiere a instrumentos económicos y financieros para promover la adopción de cambio en los patrones de producción y consumo insostenibles. ${ }^{31}$

De lo dicho hasta aquí, podemos afirmar que el Mercosur cuenta con un marco jurídico que le permitiría crear un fondo ambiental, con órganos especializados para la formulación y desarrollo de esta iniciativa y con una voluntad política, al menos declarada, en tal sentido. Tal vez haya llegado el momento de iniciar las acciones conducentes a la crea-

30 Dec. 26/07 CMC.

31 En ese marco, se encuentra en ejecución el acuerdo de cooperación técnica con la Unión Europea: "Apoyo a la Profundización del Proceso de Integración Económica y Desarrollo Sostenible del Mercosur (Econormas Mercosur). Su objetivo general es mejorar la calidad y seguridad de los productos del Mercosur y fortalecer su capacidad de conciliar el crecimiento de la actividad económica y comercial con la gestión sostenible de los recursos y el fortalecimiento de la protección ambiental. Para ello trabaja en 4 líneas de acción integradas y complementarias. Véase Convenio núm. DCI-ALA 2009/19707. 
ción de un instrumento financiero necesario para profundizar la dimensión ambiental del proceso en marcha.

\section{El Fondo para la Convergencia Estructural del Mercosur (Focem)}

El Focem es el primer fondo estructural del Mercosur. ${ }^{32}$ Fue creado por mandato del CMC, que estableció un grupo de alto nivel, con el fin de identificar iniciativas y programas que promovieran la competitividad de los países del bloque y su convergencia estructural, así como señalar formas de financiamiento para tal objetivo. ${ }^{33}$

Sin lugar a dudas, la creación del Focem constituyó un salto cualitativo en el proceso de integración. Según Monsanto: "La declaración de la necesidad de establecer fondos estructurales en el Mercosur constituye una fórmula de integración complementaria y distinta de la enunciada en su origen”, siendo ésta una manifestación del cambio axial que comenzó a notarse a partir de 2003. ${ }^{34}$

El Focem financia cuatro programas: I. Programa de Convergencia Estructural; ${ }^{35}$ II. Programa de Desarrollo de la Competitividad; ${ }^{36}$ III. Programa de Cohesión Social, ${ }^{37}$ y IV. Programa de Fortalecimiento de la Estructura Institucional y del Proceso de Integración. ${ }^{38}$

Como podrá observarse, el objetivo primordial del Focem no es la protección del medio ambiente, sino dar respuesta al problema de las

32 Establecido a través de la Dec. 45/04 del CMC, cuya integración y asignación de recursos fueron aprobados en la Dec. 18/05 del CMC.

33 En oportunidad de la XXXII Reunión Ordinaria del CMC (Río de Janeiro 2007), habiéndose adoptado todas las providencias legales, administrativas, financieras y contables para permitir la incorporación del Focem a los respectivos ordenamientos jurídicos nacionales, se estableció su entrada en operación. Para ello, mediante Dec. 8/07 el CMC aprobó los primeros once Proyectos Piloto a ser financiados por el Fondo.

34 Monsanto, A., El "nuevo Mercosur": fondos estructurales, sociedad civil y desarrollo jurídicoinstitucional, p. 7, en http:/www.portal.unr.edu.ar/internacional/elnuevomercosur.pdf.

35 Deberá contribuir al desarrollo y ajuste estructural de las economías menores, incluyendo el mejoramiento de los sistemas de integración fronteriza y de los sistemas de comunicación.

36 Tiene por finalidad contribuir a la competitividad de las producciones del Mercosur.

37 Cuyo objeto es contribuir al desarrollo social, en particular, en las zonas de frontera y podrá incluir proyectos de interés comunitario en áreas de salud, pobreza y desempleo.

38 Su objeto es el mejoramiento de la estructura institucional del Mercosur y su eventual desarrollo. 
asimetrías. No obstante, un proyecto ambiental puede calificar en algunos de los programas y, de hecho, algunas de las propuestas aprobadas se vinculan a un mejor uso de los recursos y gestión de problemas ambientales. ${ }^{39}$

Además, dado el carácter transversal de la cuestión ambiental, la misma debe estar presente en la formulación y puesta en práctica de los proyectos, ya que existe una suerte de "cláusula ambiental" entre los requisitos de admisibilidad. ${ }^{40}$

Aún aceptando las limitaciones del Focem en razón de su objeto, la experiencia de este primer fondo estructural constituye un valioso acervo a considerar, ya sea para tomarlo como modelo para la creación de un mecanismo específico ambiental o, simplemente, para proponer una reforma que permita la inclusión de un nuevo programa a financiar.

\section{A. Perfil del FOCEM}

\section{a. Reglamento}

El modo de funcionamiento del Focem está establecido en su Reglamento, ${ }^{41}$ que aborda en un solo instrumento todos los aspectos relativos a la integración y uso de los recursos, estructura institucional, programas a ser financiados, condiciones de elegibilidad de los proyectos, ciclo de aprobación, ejecución, seguimiento y monitoreo.

39 Entre los proyectos relacionados con cuestiones ambientales pueden señalarse: "Desarrollo de Capacidades e Infraestructura para Clasificadores Informales de Residuos Urbanos en Localidades del Interior del Uruguay", "Intervenciones Múltiples en Asentamientos Ubicados en Territorios de Frontera con Situaciones de Extrema Pobreza y Emergencia Sanitaria, Ambiental y Hábitat", "Programa de Acción Mercosur Libre de Fiebre Aftosa", "Construcción y mejoramiento de sistemas de agua potable y saneamiento básico en pequeñas comunidades rurales e indígenas del país Paraguay" y "Mercosur YPORA-Promoción de acceso al agua potable y saneamiento básico en comunidades en situación de pobreza y extrema pobreza Paraguay".

40 Cfr. artículo 38, inciso 8, del Reglamento del Focem, para que un proyecto pueda ser financiado debe optimizar la utilización de los recursos naturales y prever las acciones de mitigación de los daños ambientales que pudiera provocar en su área de influencia directa.

41 El primer Reglamento del Focem fue aprobado mediante Dec. 24/05 del CMC y prorrogado por Dec.15/09 del CMC. El actual Reglamento, cuya vigencia es de 4 años, fue aprobado mediante Dec. 1/10 del CMC. 
A nuestro criterio, el reglamento Focem cubre todos los aspectos centrales y resulta de fácil interpretación. A través de los primeros años de actividad observamos que este fondo puede constituir un instrumento eficaz para reducir el problema de las asimetrías de los Estados.

Puede observarse, no obstante, que en términos reales de integración, quizás reportarían más beneficios proyectos regionales que involucraran a los cuatro Estados miembros plenos del bloque. ${ }^{42}$

\section{b. Integración y distribución de los recursos}

La característica más importante del FOCEM está vinculada a la integración de los aportes de los Estados Partes y a la pauta acordada para el reparto de esos recursos a través de los proyectos que se concreten. La asignación de los fondos y su reparto está relacionada con el tamaño de sus economías: la mayor aporta más y recibe menos y viceversa. ${ }^{43}$

Este fondo se integra con aportes no reembolsables efectuados por los Estados parte de la siguiente manera: 27\% provenientes de Argentina; 70\% de Brasil; 1\% de Paraguay, y el 2\% restante de Uruguay, alcanzando un monto total de 100 millones de dólares anuales.

Los recursos se distribuyen de manera proporcional a las necesidades de los sectores o regiones más sensibles, en los siguientes porcentajes: Paraguay 48\%, Uruguay 32\%, Argentina y Brasil 10\% cada uno. En los casos de proyectos pluriestatales debe especificarse cómo se afectarán las cuotas de distribución de recursos de cada uno de los Estados partes participantes en el mismo. ${ }^{44}$

La modalidad descrita se justifica por el objeto para el cual fue creado este fondo (tratamiento de las asimetrías), pero debería ser revisado para un mecanismo que financie proyectos ambientales. En ese caso,

42 A la fecha han sido aprobados 35 proyectos, de los cuales son beneficiarios: Argentina (3), Brasil (4), Paraguay (16), Uruguay (8), pluriestatal (1) y para el fortalecimiento institucional (3). Es decir que, sin considerar los proyectos de fortalecimiento institucional presentados por la Secretaría del Mercosur, sólo se ha admitido un emprendimiento regional, el proyecto: "Programa de Acción Mercosur Libre de Aftosa-PAMA" (Dec. CMC 8/07). Para conocer los proyectos aprobados, véase http: / / www.mercosur.int/focem/.

43 Czar de Zalduendo, S., "Panorama Actual del Mercosur: ¿Meseta o Pendiente abajo?" en La Unión Europea y el Mercosur a 50 años de la firma de los Tratados de Roma, Buenos Aires, La Ley, 2008, p.23.

44 Véase Dec. 18/05 del CMC. 
podrán tenerse en cuenta diversas variables: asignaciones por país y por áreas focales, extensión de superficie con valor ambiental, mayor grado de vulnerabilidad a los cambios e incluso a los impactos ocasionados por medidas de mitigación; prevención de conflictos en aéreas de ecosistemas compartidos, compromisos internacionales asumidos por los Estados parte, entre otros. ${ }^{45}$

\section{c. Estructura institucional}

En el ciclo de formulación, aprobación y ejecución de los proyectos, intervienen el GMC y el CMC junto con varios ámbitos administrativos.

En cada uno de los Estados miembros existe una Unidad Técnica Nacional Focem (UTNF) que es la encargada de formular, presentar y ejecutar los proyectos. La gestión completa de todo proyecto financiado por este Fondo es responsabilidad del Estado beneficiario a través de la UTNF.

La Comisión de Representantes Permanentes del Mercosur (CRPM), que trabaja asistida por los representantes de cada Estado, recibe la propuesta y analiza si cumple con las condiciones de admisibilidad. En caso de resolución favorable la remite a la Unidad Técnica FOCEM (UTF), que es la instancia técnica para la evaluación y seguimiento de la ejecución de los proyectos.

La UTF, que funciona en la Secretaría del Mercosur y está integrada por el coordinador ejecutivo y funcionarios técnicos y administrativos, ${ }^{46}$ emitirá un dictamen técnico a la CRPM.

Recibido el informe por la CRPM, ésta preparará su propio dictamen, que incluirá cada uno de los proyectos considerados viables, para su consideración por el GMC.

Finalmente, el GMC remitirá al CMC el paquete de los proyectos que estén en condiciones técnicas de ser aprobados y, en ese caso, este último asignará los recursos correspondientes.

45 Esto pone de relieve, una vez más, que las cuestiones ambientales exigen inexorablemente un abordaje interdisciplinario.

46 La estructura de la UTF ha sido definida mediante Dec.24/10 del CMC, en tanto que la estructura salarial y las reglas y procedimientos específicos para la selección y contratación de su personal, fueron fijadas a través de la Res. 49/10 del GMC. 
El Reglamento establece los plazos máximos en que debe cumplirse cada una de las etapas.

Tal como señala Czar de Zalduendo, en todo el círculo se refleja una conducta tradicional del Mercosur que es la poca disposición de los países para delegar funciones, así como la resistencia a incurrir en gastos comunitarios para el mantenimiento de la estructura institucional. ${ }^{47}$

\section{d. Admisibilidad de los proyectos}

Para que un proyecto pueda ser aprobado deberá ajustarse a alguno de los cuatro programas referidos con anterioridad, debe estar propuesto y ejecutado bajo la responsabilidad del sector público de uno o más de los Estados parte (la excepción son los proyectos relativos al Programa de Fortalecimiento Institucional del Proceso de Integración, que también pueden ser propuestos por la Secretaría del Mercosur ${ }^{48}$ y los pluriestatales que pueden ser propuestos por los órganos de la estructura institucional del Mercosur), ${ }^{49}$ deben tener gastos elegibles ${ }^{50}$ y no elegibles ${ }^{51}$ que alcancen un monto superior o igual a los US\$ $500000 \mathrm{y}$ una tasa interna de retorno socioeconómico mayor que la tasa mínima de rentabilidad social.

Cabe destacar que los Estados parte deberán solventar al menos el $15 \%$ de los gastos elegibles de los proyectos de los que sean beneficiarios y la totalidad de los gastos no elegibles. ${ }^{52}$

Entendemos que el reglamento establece una serie razonable de requisitos sustanciales y formales de admisibilidad, que guardan relación con el objeto perseguido por el fondo. La exigencia de iniciativa na-

47 Czar de Zalduendo, S., op. cit., p. 25.

48 Artículo 48 inciso 2, del Reglamento Focem.

49 Artículo 39 del Reglamento Focem.

50 Son gastos elegibles aquellos inherentes al proyecto, es decir que sólo se producen si el proyecto se ejecuta, y verificables en forma conclusiva.

51 Los gastos no elegibles, entendidos como aquellos que no son propios del proyecto, figuran en el artículo 46 del Reglamento Focem. Entre ellos: compra de inmuebles, adquisición y amortización de bienes de capital usados, gastos financieros, pago de impuestos a favor del propio Estado beneficiario.

52 Artículo 47 del Reglamento Focem. 
cional resguarda el principio de la soberanía estatal sobre sus recursos naturales y en el diseño de sus propias políticas ambientales.

\section{ReFLEXIONES FINALES. CURSOS DE ACCIÓN}

La protección del medio ambiente en su concepción actual, basada en una visión global de los problemas y en el paradigma del desarrollo sustentable, es una de las dimensiones clave en todo proceso de integración regional.

En ese contexto, los fondos ambientales de carácter regional constituyen una herramienta idónea para hacer posible la cooperación internacional e, incluso, profundizar los procesos de integración.

En el caso del Mercosur, el SGT6, dada su especialidad y experiencia adquirida en los últimos años, podría abocarse a la formulación y propuesta de un Fondo de Implementación de Proyectos Ambientales.

En tal sentido, la estructura y lecciones aprendidas del funcionamiento del Focem ofrecen un valioso acervo mercosureño que puede ser capitalizado.

Es posible, entonces, pensar al menos en dos alternativas: a) crear un instrumento específico tomando como modelo al Focem, en lo que sea pertinente. Debería considerarse la experiencia de otros fondos ambientales internacionales, en particular el FMAM en el plano universal, y el LIFE en el ámbito comunitario europeo, o b) proponer una adenda al Reglamento del Focem, para incluir un nuevo programa a cofinanciar (Programa V.- Medio Ambiente y Desarrollo Sustentable), introduciendo previsiones específicas para este componente.

Para ambas posibilidades será preciso definir las áreas temáticas o focales a financiar, atendiendo a las prioridades nacionales y regionales dentro de un enfoque sistémico, así como los compromisos internacionales en materia ambiental asumidos por los miembros del bloque.

También deberá acordarse un sistema de asignación de recursos diferente al del Focem en su esquema actual, a cuyo fin resultará imprescindible contar con indicadores ambientales nacionales y regionales.

Todo mecanismo que financie proyectos ambientales debe reposar en una gestión transparente y sobre criterios de elegibilidad claros que 
se funden en una sólida base científica. Debe, asimismo, conciliar el respeto de la soberanía estatal con un enfoque sistémico del ambiente, y permitir la efectiva participación de los destinatarios directos de los proyectos, ya que esto contribuirá a una mayor base de consenso y una profundización del proceso de integración.

América de Sur es un sub continente privilegiado por sus inconmensurables riquezas naturales; gracias a sus caudalosos ríos y acuíferos, es, quizás, la reserva de agua dulce más grande del mundo; es uno de los pulmones del planeta con la mayor extensión de selvas y bosques lluviosos, y está bendecido por dos océanos que lo abrazan. Querer a esta tierra es cuidarla, es conciliar la economía y el comercio con la protección del ambiente. Ya no se trata de un desafío sino de una obligación de los líderes de la región y la integración se ofrece como uno de los caminos posibles. Es tiempo de poner manos a la obra y llenar de contenido la retórica de bellos discursos.

\section{BIBLIOGRAFÍA}

Barboza, Julio, Derecho internacional público, 2a. ed., Buenos Aires, Zavalia, 2001.

CASSESE, Antonio, International Law, 2a. ed., Nueva York, Oxford University Press, 2005.

CEPAL (Comisión Económica para América Latina), El desarrollo sustentable: transformación productiva, equidad y medio ambiente, Santiago de Chile, Naciones Unidas, 1991.

Cienfuegos Mateo, Manuel, “¿Es la Unión de Naciones Sudamericana un proyecto viable?”, Mercosur y Unión Europea, Córdoba, Lerner, 2008.

CORIA, Silvia, Integración, desarrollo sustentable y medio ambiente, Buenos Aires, Ciudad Argentina, 1997.

CZar de Zalduendo, Susana, "Panorama Actual del Mercosur: ¿Meseta o Pendiente abajo?”, La Unión Europea y el Mercosur: a 50 años de la firma de los Tratados de Roma, Buenos Aires, La Ley, 2008.

DAillier, Patrick y Pellet, Alain, Droit International Public, 7a. ed., París, L.G.D.J., 2002. 
De Miguel Perales, Carlos, La responsabilidad civil por daños al medio ambiente, Madrid, Civitas, 1993.

DeviA, Leila, "La política ambiental en el marco del Tratado de Asunción”, Mercosur y Medio Ambiente, Buenos Aires, Ciudad Argentina, 2000.

Di PAOlA, María, "El daño ambiental y la sustentabilidad”, Ambiente, derecho y sustentabilidad, Buenos Aires, La Ley, 2000.

GRAJATE, Rita, "Hacia la construcción de la Unasur. Institucionalidad y Convergencia”, Mercosur y Unión Europea, Córdoba, Lerner, 2008.

LACIAR, Mirta, Medio ambiente y desarrollo sustentable. Los desafíos del Mercosur, Buenos Aires-Madrid, Ciudad Argentina, 2003.

Monsanto, Alberto, El "nuevo Mercosur", Fondos estructurales, sociedad civil y desarrollo jurídico-institucional, disponible en: http: / www.portal. unr.edu.ar/internacional / elnuevomercosur.pdf.

Parejo Alfonso, Luciano et al., Derecho medioambiental de la Unión Europea, Mc Graw-Hill, Madrid, 1996.

Pastorino, Leonardo, El Daño al Ambiente, Argentina, Lexis Nexis, 2005.

Plaza Martín, Carmen, Derecho ambiental de la Unión Europea, Madrid, Tirant Lo Blanch, 2005.

Remond-Gouilloud, Martine, Du droit de détruire, París, PUF, 1989. , "Los fonds d'índemnisation et le préjudice écologique", Le dommage écologique, París, Société Française pour le Droit de l'Environneme, 1992.

RYAN, Daniel, "Mercosur y ambiente”, Ambiente, derecho y sustentabilidad, Buenos Aires, La Ley, 2000.

SANDS, Philippe, Principles of International Environmental Law, 2a. ed., Cambridge, Cambridge University Press, 2003.

SAyd (Secretaría de Ambiente y Desarrollo Sustentable de la NACIÓN) y GTZ (DEUSTCHE GeSELLSCHAFT FÜr TECHNisChe ZuSAMmeNARBEIT), Gestión ambiental y producción más limpia en el Mercosur. Proyecto "Competitividad y Medio Ambiente" en la Argentina. Cinco años con la Cooperación Alemana.

STRONG, Maurice, "Se promueven las inversiones en el desarrollo sostenible”, Revista Nuestro Planeta, Naciones Unidas, Extra, 1997. 
TÖPfER, Klaus, "Fondo para el Medio Ambiente Mundial. Un interés común”, Revista Nuestro Planeta, Naciones Unidas, Extra, 1997

VernetTI, Ana, "Medio ambiente: los fondos ambientales como mecanismos financieros de recomposición“, Revista Doctrina Judicial, Buenos Aires, 2001-3.

, "Camino al desarrollo sustentable", Enfoques Ambientales, Mar del Plata, Suarez, 2006. 